\title{
Simultaneous temporal superresolution and denoising for cardiac fluorescence microscopy
}

\author{
Kevin G. Chan, Student Member, IEEE, Sebastian J. Streichan, Le A. Trinh, and Michael Liebling, Member, IEEE
}

\begin{abstract}
Due to low light emission of fluorescent samples, live fluorescence microscopy imposes a tradeoff between spatiotemporal resolution and signal-to-noise ratio. This can result in images and videos containing motion blur or Poisson-type shot noise, depending on the settings used during acquisition. Here, we propose an algorithm to simultaneously denoise and temporally super-resolve movies of repeating microscopic processes that is compatible with any conventional microscopy setup that can achieve imaging at a rate of at least twice that of the fundamental frequency of the process (above 4 frames per second for a $2 \mathrm{~Hz}$ process). Our method combines low temporal resolution frames from multiple cycles of a repeating process to reconstruct a denoised, higher temporal resolution image sequence which is the solution to a linear program that maximizes the consistency of the reconstruction with the measurements, under a regularization constraint. This paper describes, in particular, a parallelizable superresolution reconstruction algorithm and demonstrates its application to live cardiac fluorescence microscopy. Using our method, we experimentally show temporal resolution improvement by a factor of 1.6, resulting in a visible reduction of motion blur in both on-sample and off-sample frames.
\end{abstract}

Index Terms-Temporal superresolution, motion blur, image denoising, image reconstruction, fluorescence microscopy.

\section{INTRODUCTION}

Superresolution microscopy has recently gained popularity with the development of new techniques, such as stimulated emission depletion microscopy (STED) [1], [2] and structured illumination microscopy (SIM) [3], [4]. These superresolution techniques, along with localization techniques [5] such as PALM [6] and STORM [7], allow for imaging with resolution finer than that set by the optical diffraction limit. While much of the research has focused on improving spatial resolution, temporal resolution in microscopy has received considerably less attention despite it being a significant issue in live fluorescence imaging. And although a number of fast imaging methods have been proposed [8]-[10], the fact still remains that live fluorescence imaging of dynamic samples imposes a tradeoff between spatio-temporal resolution and signal-to-noise ratio during acquisition [11]. Temporal resolution in fluorescence microscopy is limited by the fluorophore emission rate, which imposes that the camera integration time must be long enough

K. G. Chan and M. Liebling are with the Department of Electrical and Computer Engineering, University of California, Santa Barbara, CA, 93106, USA.

M. Liebling is with the Idiap Research Institute, 1920 Martigny, Switzerland (email: michael.liebling@idiap.ch).

S. J. Streichan is with the Kavli Institute for Theoretical Physics, University of California, Santa Barbara, CA, 93106, USA.

L. A. Trinh is with the Molecular and Computational Biology and Translational Imaging Center, University of Southern California, Los Angeles, CA 90089 USA. to acquire enough photons for an acceptable signal-to-noise ratio. Unfortunately, when imaging rapid, dynamic processes, a long integration time results in motion blur, and a low sampling rate can result in temporal aliasing. Using a higher sampling rate and a shorter exposure time can reduce motion blur and temporal aliasing, but will also decrease the signalto-noise ratio. Furthermore, increasing the sampling rate and decreasing the exposure time is not always possible, since cameras designed for fluorescence microscopy have an upper limit on their frame rate (typically between 30-100 frames per second) and a lower limit on integration time (often on the order of milliseconds). We wish to image rapid, dynamic processes (such as the beating heart in developing animal embryos) with both high temporal resolution and a high signalto-noise ratio. Since this is not possible using a hardware solution exclusively, we propose to overcome this tradeoff by implementing a computational method that simultaneously improves both temporal resolution and signal-to-noise ratio, at the cost of taking multiple acquisitions of the same dynamic process.

Our approach differs from the existing methods that have been proposed for temporal superresolution, whose main features we summarize hereafter. Bub et al. proposed a temporal pixel multiplexing method for microscopy to achieve temporal superresolution by offsetting pixel exposure times during a single frame capture [12]. However, this came at the cost of spatial resolution, and it required a modified camera setup with multiplexed pixels. In a related work, Reddy et al. proposed a novel camera architecture with programmable, multiplexed pixels [13]. This method was able to recover a high-speed image sequence at full spatial resolution by exploiting the spatio-temporal redundancy in videos. However, the method again required a modified camera that may not be readily available to most microscopy labs. Additionally, the method assumed that spatially moving objects remain at a constant brightness along the time dimension, which may not be true in fluorescence biomicroscopy. Other methods, such as the staggered exposure approach by Shechtman et al. [14] and the coded sampling work by Agrawal et al. [15] used multiple synchronized cameras, each with different temporal sampling patterns, to achieve temporal superresolution. However, such approaches are not well-suited to fluorescence microscopy, since they would divide the already low photon count between multiple cameras. Related to [15], Veeraraghavan et al. proposed a coded strobing method using a single camera to image high-speed periodic events [16]. However, strobing and pulsed illumination methods, while able to reduce motion blur for high-speed imaging, require precisely-controlled, high 
intensity illumination [17]. When imaging live fluorescent samples, using high intensity illumination can lead to fluorophore saturation, photodamage, and photobleaching [18], [19].

We are interested in imaging the dynamics of the beating heart in fluorescence under low illumination intensity, at full spatial resolution, at high frame rates, with a high signalto-noise ratio, and with hardware commonly found in most microscopy labs. To accomplish this, we take advantage of the repetitive motion of the heart to acquire a long, low temporal resolution image sequence containing multiple heart cycles. We then combine these multiple low temporal resolution cycles into a higher temporal resolution version of a single heart cycle. While we focus on imaging the heart, our method is designed to be applicable to imaging any repeating process.

Our approach is related to the spatial superresolution method of, for example, Sroubek et al. [20], Farsiu et al. [21], and Ben-Ezra et al. [22], where several low spatial resolution images with translational shifts were combined into a high spatial resolution image. Here, we instead reconstruct a temporal superresolution sequence from multiple low temporal resolution image sequences. Our reconstruction approach leverages two key ingredients: (i) availability of multiple low resolution measurements and (ii) knowledge about the imaging system (including its parameters), as specified through a well-defined forward model. Attempting to recover the high resolution sequence from a single low resolution measurement would result in a highly ill-posed inverse problem: the system is under-determined, and many different high resolution solutions could produce the same low resolution measurements. Instead, by combining multiple measurements, each collected with different acquisition parameters (i.e. temporal shifts), the problem becomes over-determined, and can be solved to yield super-resolution.

While a previous method we developed assumed uniform temporal shifts between low resolution sequences [23], here we allow for non-uniform, sub-resolution temporal warping in the acquired image sequence for repeating motions that are not strictly periodic. In addition to improving resolution, this superresolution approach is robust to noise, and the combination of multiple acquisitions has a noise reducing effect. Unlike other methods to increase the frame-rate, our approach does not require any modifications to the conventional image acquisition setup. It only requires collecting multiple temporal cycles of one's sample with an imaging rate at least twice that of the fundamental frequency of the process. We make a Fiji [24] plugin to demonstrate the superresolution reconstruction available online at [25].

This paper is organized as follows. In Section II, we present the acquisition and superresolution method. In Section III, we demonstrate the improvement this technique offers for temporal resolution and noise reduction in live cardiac microscopy. In Section IV, we summarize the advantages of our method and discuss some details to consider when using the algorithm.

\section{Methods}

Many biological processes, such as the expansion and contraction of the heart, are repeating. We take advantage of this repetition to reconstruct a single cycle with high framerate from a low frame-rate movie containing multiple cycles of our process of interest.

\section{A. Image Acquisition Model}

At every pixel (voxel) coordinate $\mathbf{x}=(x, y, z)$, we wish to reconstruct a single, high temporal resolution cycle of the sample's emitted fluorescence intensity, $I_{\text {ref }}[\mathbf{x}, n], n=0, \ldots, N_{\text {ref }}-1$, where $N_{\text {ref }}$ is the number of samples covering the duration of one cycle, and where the sample spacing $T_{\mathrm{H}}$ is sufficiently small to prevent temporal aliasing of the fundamental frequency. We assume that at a given coordinate $\mathbf{x}$, the sample's fluorescence emission intensity, $I[\mathbf{x}, n]$, at any time point $n \in \mathbb{Z}$ (not only $0 \leq n<N_{\text {ref }}$ ), is a temporally repeating signal such that $I[\mathbf{x}, n]$ is either equal (up to some additive noise) to a matching time point in $I_{\text {ref }}$, or can be interpolated from $I_{\text {ref }}$, provided that $I_{\text {ref }}$ is chosen to be a complete cycle. More specifically, using linear interpolation, our assumption translates to:

$$
\begin{aligned}
I[\mathbf{x}, n]= & (1-(w[n]-\lfloor w[n]\rfloor)) I_{\mathrm{ref}}[\mathbf{x},\lfloor w[n]\rfloor] \\
& +(w[n]-\lfloor w[n]\rfloor) I_{\mathrm{ref}}[\mathbf{x},\lceil w[n]\rceil] \\
& +v_{s}[\mathbf{x}, n]
\end{aligned}
$$

where $w[n]$ is the real-valued "index" in $I_{\text {ref }}$ that corresponds to $I[\mathbf{x}, n]\left(0 \leq w[n]<N_{\text {ref }}\right)$, and $v_{s}[\mathbf{x}, n]$ is an additive noise term.

Our approach consists of acquiring a long sequence $\hat{I}[\mathbf{x}, n], n=0, \ldots, N-1$ containing $K>1$ cycles of our repeating process with a larger sample spacing $T_{\mathrm{L}}\left(T_{\mathrm{L}}>T_{\mathrm{H}}\right)$, and we aim at combining information from all $K$ cycles to obtain an estimate of the reference sequence $I_{\text {ref }}[\mathbf{x}, n]$ with the desired sample spacing $T_{\mathrm{H}}$. We further assume that the ratio between sampling steps, $\frac{T_{\mathrm{L}}}{T_{\mathrm{H}}}=\frac{p}{q}$, is a rational number, and we assume that the number of measured periods is more than the sampling ratio (i.e. $1<\frac{p}{q}<K$ ) so that the problem is overdetermined.

The measured sequence can be expressed as a discrete convolution of an upsampled (by insertion of $q-1$ zeros between samples) version of the high-resolution sequence with the system's temporal impulse response $h$, followed by a decimation by $p$. Specifically,

$$
\hat{I}[\mathbf{x}, n]=\left((I[\mathbf{x}, \cdot])_{\uparrow q} * h\right)_{\downarrow p}[\mathbf{x}, n]+v_{m}[\mathbf{x}, n],
$$

where $v_{m}[\mathbf{x}, n]$ represents additive measurement noise, and $h$ represents the rectangular filter,

$$
h[n]=\left\{\begin{array}{ll}
\frac{1}{N_{\text {int }}} & 0 \leq n<N_{\text {int }} \\
0 & \text { otherwise }
\end{array},\right.
$$

modeling the system's temporal integration time $T_{\text {int }}$ and integration window width, $N_{\mathrm{int}}=\operatorname{round}\left(\frac{T_{\mathrm{int}}}{T_{\mathrm{L}}} \frac{p}{q}\right)$. This convolution and temporal warping is shown in Figure 1, where each measured sample, $\hat{I}[\mathbf{x}, n]$, is an integration of the ideal reference signal, $I_{\text {ref }}[\mathbf{x}, n]$, over some time window (denoted by the dark rectangles). Since the measured signal repeats, some samples will correspond to overlapping windows in the ideal signal that are slightly shifted by a fractional offset. 


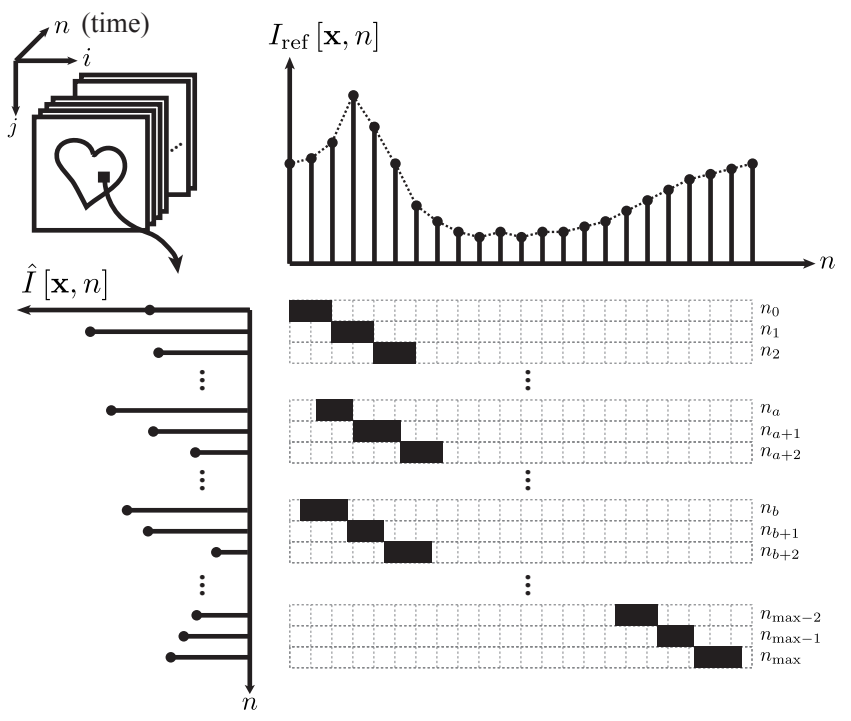

Fig. 1. A temporally repeating process (such as the cardiac cycle) is imaged over multiple cycles using a long exposure time and a low frame rate to produce $\hat{I}[\mathbf{x}, n]$. Each acquired frame corresponds to a finite time window (denoted by the dark rectangles) of the underlying model signal, $I_{\mathrm{ref}}[\mathbf{x}, n]$. Although the camera frame-rate $1 / T_{\mathrm{L}}$ and integration time $T_{\text {int }}$ are always kept constant, the measured signal may vary temporally, compared to the model signal. Therefore, a frame in one cycle may correspond to a different duration of the underlying signal compared to a similar frame in a different cycle.

Using discrete matrix-vector notation similar to that used in [20], we can also express our forward model at pixel location $\mathbf{x}=(x, y, z)$ as

$$
\mathbf{g}_{\mathbf{x}}=\mathbf{D}_{p} \mathbf{H U}_{q} \mathbf{S f}_{\mathbf{x}}+\mathbf{v}_{\mathbf{x}}
$$

where

$$
\begin{gathered}
\mathbf{g}_{\mathbf{x}}=(\hat{I}[\mathbf{x}, 0], \ldots, \hat{I}[\mathbf{x}, N-1])^{\top}, \\
\mathbf{f}_{\mathbf{x}}=\left(I_{\mathrm{ref}}[\mathbf{x}, 0], \ldots, I_{\text {ref }}\left[\mathbf{x}, N_{\text {ref }}-1\right]\right)^{\top},
\end{gathered}
$$

$\top$ denoting transposition, and $\mathbf{D}_{p}$ is an $N \times N p$ matrix that downsamples by $p, \mathbf{H}$ is an $N p \times N p$ circulant matrix with shifted versions of the filter $h[n]$ as its rows, $\mathbf{U}_{q}$ is an $N p \times N p / q$ matrix that upsamples by $q, \mathbf{S}$ is an $N p / q \times N_{\text {ref }}$ matrix with elements

$$
\mathbf{S}_{i j}= \begin{cases}1-(w[i]-\lfloor w[i]\rfloor) & j=\lfloor w[i]\rfloor \\ w[i]-\lfloor w[i]\rfloor & j=\lceil w[i]\rceil \\ 0 & \text { otherwise }\end{cases}
$$

that rearranges and interpolates the high-resolution reference cycle $\mathbf{f}_{\mathbf{x}}$ according to sub-frame positions $w[n]$ (unknown in practice), and $\mathbf{v}_{\mathbf{x}}$ is an additive noise vector that incorporates both $v_{m}[\mathbf{x}, n]$ and $v_{s}[\mathbf{x}, n]$.

\section{B. Sub-resolution Temporal Registration}

The first task to invert Equation (4) is estimating the best matching sub-frame indices, $w[n]$, for each time point $n=0, \ldots, N-1$. To do this, we first define a new set of sub-frame indices in the low-resolution signal,

$$
w_{\ell}[n]=\frac{q}{p} w[n]
$$

which we split into a large, whole-sample integer shift $\bar{w}_{\ell}[n]$ and a smaller sub-sample shift $\Delta_{n}$,

$$
w_{\ell}[n]=\bar{w}_{\ell}[n]+\Delta_{n},
$$

where $\left|\Delta_{n}\right|<1$. We then use a two-step process to separately estimate $\bar{w}_{\ell}[n]$ and $\Delta_{n}$ that is similar to the approach used in [26], with the exception that we explicitly estimate the subsample shift.

We first identify a low-resolution reference cycle $\hat{I}_{\text {ref }}[\mathbf{x}, m], m=0, \ldots,\left\lfloor\frac{q}{p} N_{\text {ref }}\right\rfloor-1$ from the long sequence $\hat{I}[\mathbf{x}, n]$. For simplicity, we consider the first $M=\left\lfloor\frac{q}{p} N_{\text {ref }}\right\rfloor$ time points in $\hat{I}[\mathbf{x}, n]$ to be our reference cycle $\hat{I}_{\text {ref }}[\mathbf{x}, m]$. In practice, the reference cycle can be user-defined by its first and last frame, determined automatically by taking the $M$ first frames (with $M$ an estimate of the cardiac frequency obtained from the peak frequency component in the Fourier transform of the entire signal), or a combination of both (user-adjustment following automatic period estimation). While the sub-frame accuracy of the reference sequence is not necessary for successful super-resolution, the assumption in Equation (1), which states that each time point in the long sequence can be obtained by interpolating two frames from the reference cycle, should not be violated.

To determine the whole-sample shift $\bar{w}_{\ell}[n]$, we find the best match $\hat{I}_{\text {ref }}\left[\mathbf{x}, \bar{w}_{\ell}[n]\right]$ to $\hat{I}[\mathbf{x}, n]$. Specifically, we use a dynamic programming synchronization algorithm [27] to find $\tilde{w}[n] \in \mathbb{Z}$ for $n=0, \ldots, N-1$, such that

$$
\mathcal{Q}=\sum_{n=0}^{N-1} \sum_{\mathbf{x}}\left|\hat{I}[\mathbf{x}, n]-\hat{I}_{\text {ref }}[\mathbf{x},(\tilde{w}[n] \bmod M)]\right|
$$

is minimized with respect to $\tilde{w}[n]$, under the constraint that

$$
w_{\min } \leq \tilde{w}[n]-\tilde{w}[n-1] \leq w_{\max },
$$

where mod denotes the modulo operator, $M=\left\lfloor\frac{q}{p} N_{\text {ref }}\right\rfloor$, and $w_{\min }$ and $w_{\max }$ are positive integers $\left(0<w_{\min } \leq w_{\max }\right)$ that allow for nonuniform temporal warping, restrict $\tilde{w}[n]$ to be monotonically increasing, and limit excessive dilation. In practice, $w_{\min }$ and $w_{\max }$ can be used to restrict the search space, and thereby reduce the computational burden, when bounds on the variability in heartbeat rhythm can be estimated. The whole-sample shift is then given by

$$
\bar{w}_{\ell}[n]=\tilde{w}[n] \bmod M .
$$

To determine the sub-sample shift $\Delta_{n}$, we approximate the reference signal at spatial location $\mathbf{x}$ and sub-integer index $w_{\ell}[n]=\bar{w}_{\ell}[n]+\Delta_{n}$ with a first order Taylor series and a finite difference approximation to the derivative,

$$
\begin{aligned}
\hat{I}[\mathbf{x}, n] & \approx \hat{I}_{\text {ref }}\left[\mathbf{x}, \bar{w}_{\ell}[n]\right]+ \\
& \Delta_{n}\left(\hat{I}_{\mathrm{ref}}\left[\mathbf{x}, \bar{w}_{\ell}[n+1]\right]-\hat{I}_{\text {ref }}\left[\mathbf{x}, \bar{w}_{\ell}[n]\right]\right) .
\end{aligned}
$$

Using a least-squares approach, we take all spatial locations $\mathbf{x}$ into account to find

$$
\begin{aligned}
\Delta_{n}= & \underset{s}{\arg \min } \sum_{\mathbf{x}}\left(\left(\hat{I}[\mathbf{x}, n]-\hat{I}_{\text {ref }}\left[\mathbf{x}, \bar{w}_{\ell}[n]\right]\right)\right. \\
& \left.-\left(\hat{I}_{\text {ref }}\left[\mathbf{x}, \bar{w}_{\ell}[n+1]\right]-\hat{I}_{\text {ref }}\left[\mathbf{x}, \bar{w}_{\ell}[n]\right]\right) s\right)^{2} .
\end{aligned}
$$


This is a reverse interpolation problem using a piecewise linear approximation to the underlying function $\hat{I}_{\text {ref }}[\mathbf{x}, n]$. Instead of using linear interpolation to find the value of the function at a given location, we wish to find the location at which the function takes on a given value, assuming that the function is piecewise linear. In practice, we use a higher order approximation (such as the cubic approximation described in Appendix A) rather than a linear approximation to the underlying function.

Once we obtain $\bar{w}_{\ell}[n]$ and $\Delta_{n}$, we can determine the best matching sub-integer reference index

$$
w[n]=\frac{p}{q}\left(\bar{w}_{\ell}[n]+\Delta_{n}\right)
$$

and populate the matrix $\mathbf{S}$ in Equation (7).

\section{Superresolution Reconstruction}

The task of superresolution reconstruction is an inverse problem to recover the unknown $\mathbf{f}_{\mathbf{x}}$ from the measured $g_{\mathbf{x}}$. We solve this independently at each spatial location $\mathbf{x}=(x, y, z)$ by minimizing an $\ell_{1}$ cost function,

$$
\hat{\mathbf{f}}_{\mathbf{x}}(\lambda)=\underset{\mathbf{f}_{\mathbf{x}}}{\arg \min }\left\|\mathbf{D}_{p} \mathbf{H U}_{q} \mathbf{S f}_{\mathbf{x}}-\mathbf{g}_{\mathbf{x}}\right\|_{1}+\lambda\left\|\boldsymbol{\Gamma f}_{\mathbf{x}}\right\|_{1},
$$

where $\lambda$ is a regularization weighting constant, and $\boldsymbol{\Gamma}$ is a Tikhonov regularization second-order difference operator that is chosen to favor temporally smooth solutions,

$$
\boldsymbol{\Gamma}=\left[\begin{array}{rrrrrr}
2 & -1 & 0 & \ldots & 0 & -1 \\
-1 & 2 & -1 & \ldots & 0 & 0 \\
& & & \ddots & & \\
-1 & 0 & 0 & \ldots & -1 & 2
\end{array}\right]
$$

and all other matrices are given by the forward imaging model in Equation (4). This is a strictly one-dimensional temporal reconstruction problem, and we solve this independently at each pixel without any spatial constraints.

The minimization in Equation (16) is equivalent to the minimization problem:

$$
\begin{aligned}
\hat{\mathbf{f}}_{\mathbf{x}}=\underset{\mathbf{f}_{\mathbf{x}}, \tilde{\mathbf{y}}}{\arg \min } \sum_{i} \tilde{\mathbf{y}}_{i} \text { s.t. } \\
\quad-\tilde{\mathbf{y}} \leq\left[\frac{\mathbf{D}_{p} \mathbf{H} \mathbf{U}_{q} \mathbf{S}}{\lambda \boldsymbol{\Gamma}}\right] \mathbf{f}_{\mathbf{x}}-\left[\frac{\mathbf{g}_{\mathbf{x}}}{\mathbf{0}}\right] \leq \tilde{\mathbf{y}},
\end{aligned}
$$

where $\tilde{\mathbf{y}}$ is a helper variable vector that bounds the data fidelity error and the regularization error. Since the doublesided inequality in Equation (18) can be rewritten as a singlesided inequality,

$$
\left[\begin{array}{c}
\mathbf{D}_{p} \mathbf{H} \mathbf{U}_{q} \mathbf{S} \\
-\mathbf{D}_{p} \mathbf{H} \mathbf{U}_{q} \mathbf{S} \\
\lambda \boldsymbol{\Gamma} \\
-\lambda \boldsymbol{\Gamma}
\end{array}\right] \mathbf{f}_{\mathbf{x}}-\tilde{\mathbf{y}} \leq\left[\begin{array}{c}
\mathbf{g}_{\mathbf{x}} \\
-\mathbf{g}_{\mathbf{x}} \\
\mathbf{0} \\
\mathbf{0}
\end{array}\right]
$$

we can pose the minimization in Equation (16) as a linear programming problem subject to an inequality constraint,

$$
\begin{aligned}
\hat{\mathbf{y}}= & \min \mathbf{c}^{\top} \mathbf{y} \\
& \text { s.t. } \mathbf{A y} \leq \mathbf{b} \\
& \text { and } \mathbf{y} \geq \mathbf{0},
\end{aligned}
$$

with the following matrix definitions:

$$
\begin{aligned}
\mathbf{c}^{\top} & =\left[\mathbf{0}_{1 \times N_{\text {ref }}} \mid \mathbf{1}_{1 \times\left(N+N_{\text {ref }}\right)}\right], \\
\mathbf{y} & =\left[\begin{array}{c}
\left(\mathbf{f}_{\mathbf{x}}\right)_{N_{\mathrm{ref}} \times 1} \\
\tilde{\mathbf{y}}_{\left(N+N_{\text {ref }}\right) \times 1}
\end{array}\right], \\
\mathbf{A} & =\left[\begin{array}{ccc}
\mathbf{D}_{p} \mathbf{H} \mathbf{U}_{q} \mathbf{S} & -\mathbf{I}_{N \times N} & \mathbf{0}_{N \times N_{\text {ref }}} \\
-\mathbf{D}_{p} \mathbf{H} \mathbf{U}_{q} \mathbf{S} & -\mathbf{I}_{N \times N} & \mathbf{0}_{N \times N_{\text {ref }}} \\
\lambda \boldsymbol{\Gamma} & \mathbf{0}_{N_{\text {ref }} \times N} & -\mathbf{I}_{N_{\text {ref }} \times N_{\text {ref }}} \\
-\lambda \boldsymbol{\Gamma} & \mathbf{0}_{N_{\text {ref }} \times N} & -\mathbf{I}_{N_{\text {ref }} \times N_{\text {ref }}}
\end{array}\right], \\
\mathbf{b} & =\left[\begin{array}{c}
\mathbf{g}_{\mathbf{x}} \\
-\mathbf{g}_{\mathbf{x}} \\
\mathbf{0}_{N_{\text {ref }} \times 1} \\
\mathbf{0}_{N_{\text {ref }} \times 1}
\end{array}\right] .
\end{aligned}
$$

We find the solution to this linear programming problem by the simplex method [28]. To reconstruct the full temporal superresolution video, we apply the optimization at each pixel location independently. We then produce the complete temporal superresolution sequence,

$$
\hat{I}_{\mathrm{SR}}[\mathbf{x}, n]=\hat{I}_{\mathrm{SR}}[x, y, z, n]=\hat{\mathbf{f}}_{\mathbf{x}}(\lambda)
$$

by assembling the solutions at all pixel locations $\mathbf{x}=(x, y, z)$. Since each pixel location can be treated independently, the computation time can be significantly reduced through parallelization on multi-core processors or multi-node clusters.

\section{Optimal value of $\lambda$}

If, for each value of $\lambda$, the energy of each of the two terms in the minimization (16) is computed separately and a locus is recorded in a plot with the two energies in the $x$ and $y$ axes, respectively, one can empirically observe that the resulting curve has the approximate shape of the letter L. The optimal choice for $\lambda$ is the corner of this L-curve [29], as it provides a good balance between the two terms of Equation (16). We find this corner by finding the value of $\lambda \in\left[\lambda_{\min }, \lambda_{\max }\right]$ that minimizes the two-group linear regression error,

$$
\begin{aligned}
\lambda_{\mathrm{opt}}=\underset{\lambda^{\star}}{\arg \min } & \sum_{\lambda=\lambda_{\min }}^{\lambda^{\star}}\left(L(\lambda)-\hat{L}_{1}(\lambda)\right)^{2} \\
& +\sum_{\lambda=\lambda^{\star}}^{\lambda_{\max }}\left(L(\lambda)-\hat{L}_{2}(\lambda)\right)^{2},
\end{aligned}
$$

where

$$
L(\lambda)=\left(\left\|\mathbf{D}_{p} \mathbf{H} \mathbf{U}_{q} \mathbf{S} \hat{\mathbf{f}}_{\mathbf{x}}(\lambda)-\mathbf{g}_{\mathbf{x}}\right\|_{1}, \lambda\left\|\boldsymbol{\Gamma} \hat{\mathbf{f}}_{\mathbf{x}}(\lambda)\right\|_{1}\right)
$$

is a point on the L-curve, and $\hat{L}_{1}, \hat{L}_{2}$ are least-squares linear regressions to $L(\lambda)$ in the ranges $\left[\lambda_{\min }, \lambda^{\star}\right]$ and $\left[\lambda^{\star}, \lambda_{\max }\right]$, respectively. The motivation behind this approach is that the L-curve's corner location is the point that best separates the L-curve into two straight-line regions. 
(a)
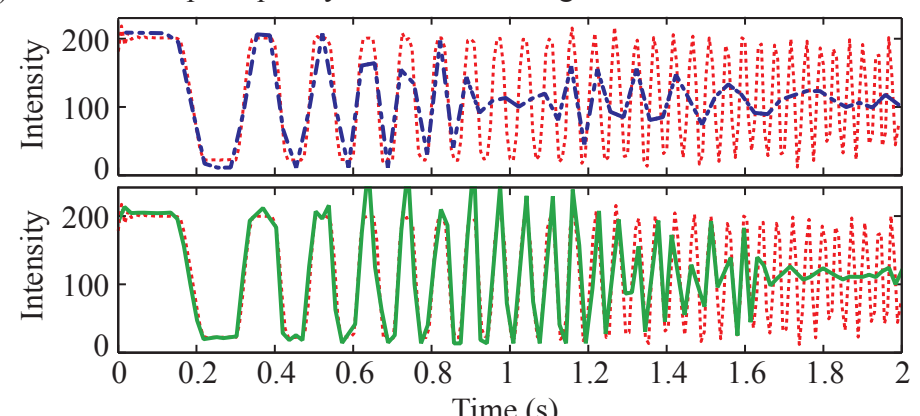

(b)

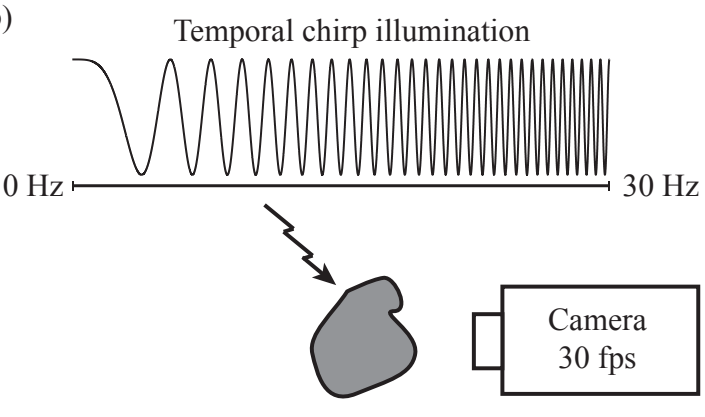

Static Object

(c)

Fourier Spectrum of Temporal Chirp

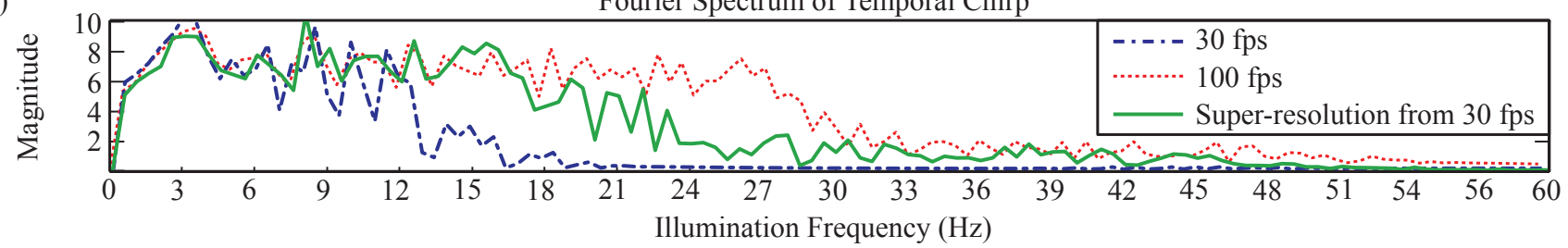

Fig. 2. We measure a static fluorescent sample's mean intensity over time while under a linear sweep-frequency (chirp) illumination from $0 \mathrm{~Hz}$ to $30 \mathrm{~Hz}$, as illustrated in (b). Sampling a linear chirp signal below the Nyquist rate results in temporal aliasing, as shown in (a: top). A non-aliased acquisition taken at $100 \mathrm{fps}$ is shown for comparison. Fourteen sequences acquired at $30 \mathrm{fps}$ are used for temporal superresolution reconstruction. The superresolution sequence, shown in (a: bottom) reveals rapid, high frequency changes in intensity that are normally lost when imaging at 30 fps. A comparison of the signals' frequency spectrum in (c) reveals a superresolution improvement in bandwidth over the 30 fps sequence by a factor of $1.6 \times$. See supplementary movie 1 for the full sequence.

\section{EXPERIMENTS}

To validate our method, we conducted both computational simulations and in vivo experiments. During the in vivo experiments, we imaged spatially static (but with a temporally varying intensity) as well as moving samples using an epi-fluorescence microscopy setup (illumination and light collection occur through the same microscope objective). To demonstrate our method with 4D imaging, we used a multiview selective plane illumination microscope [30] to image live zebrafish embryos. After acquisition, we reconstructed a full resolution, temporal superresolution video by solving the linear programming problem in Equation (20) using the cplexlp solver from the IBM CPLEX optimization package [31]. In our experiments, we assumed loose bounds on the signal's temporal warping with $w_{\min }=1$ and $w_{\max }=\frac{M}{2}$. We obtained all our $\ell_{1}$ reconstructions through independent temporal processing on a pixel-by-pixel basis without any spatial post-processing. For each $\ell_{1}$ reconstruction, unless otherwise mentioned, we determined the value of $\lambda$ by performing the reconstruction on a small, manually-selected region of interest with several different values and selecting the optimal value according to Equation (23). All experiments and procedures involving zebrafish embryos were performed following standard techniques [32] under a protocol approved by the Institutional Animal Care and Use Committee at the University of California, Santa Barbara.

\section{A. Quantifying Resolution Improvement}

To quantify the method's temporal resolution improvement experimentally, we imaged a static fluorescent sample (drawn on a glass coverslip with a Sharpie Accent Highlighter, Sanford L.P., Oak Brook, III.) illuminated by a time-varying light source. The illumination (and also the emitted fluorescence) followed a repeating temporal chirp signal, where each cycle is a sinusoid with its frequency increasing linearly from 0 $\mathrm{Hz}$ to $30 \mathrm{~Hz}$ over two seconds. We imaged the sample with a Leica DMI6000B inverted microscope and an HCX PL S-APO $20 \times / 0.50$ air objective. We acquired fourteen low temporal resolution cycles, each with a known uniform temporal shift, at 30 frames per second using a Hamamatsu ImageEM C9100-13 EM-CCD camera. EM-CCD cameras have a much higher sensitivity and gain than regular CCD cameras, and therefore are well adapted for fluorescence microscopy despite the relatively low frame rate at full resolution and full field $(512 \times 512$ pixels per frame). At 30 frames per second, the second half of the sequence (corresponding to illumination frequencies between $15 \mathrm{~Hz}$ to $30 \mathrm{~Hz}$ ) is aliased because the camera sampling rate is too slow to accurately capture the rapid flickering of the sample. Naive interpolation and resampling cannot recover these aliased high frequencies. However, by using 14 low resolution cycles (with known uniform shifts) to reconstruct a sequence with a $4 \times$ temporal magnification factor, our temporal superresolution method recovers oscillations up to $24 \mathrm{~Hz}$ (Figure 2, Supplementary movie 1). This is equivalent to a 1.6-fold increase in bandwidth, which is consistent with the theoretically-derived practical superresolution limit in [33].

We conducted further simulations to explore the relationship between the number of cycles used for reconstruction and the superresolution performance. In these simulations, we used a one-dimensional temporal chirp signal, with its frequency increasing linearly from $0 \mathrm{~Hz}$ to $150 \mathrm{~Hz}$ over one second, as the original high-resolution signal. The signal was lowpass filtered and sampled at $150 \mathrm{~Hz}$ so that frequencies above $75 \mathrm{~Hz}$ were lost. For reconstruction, we first assumed the 


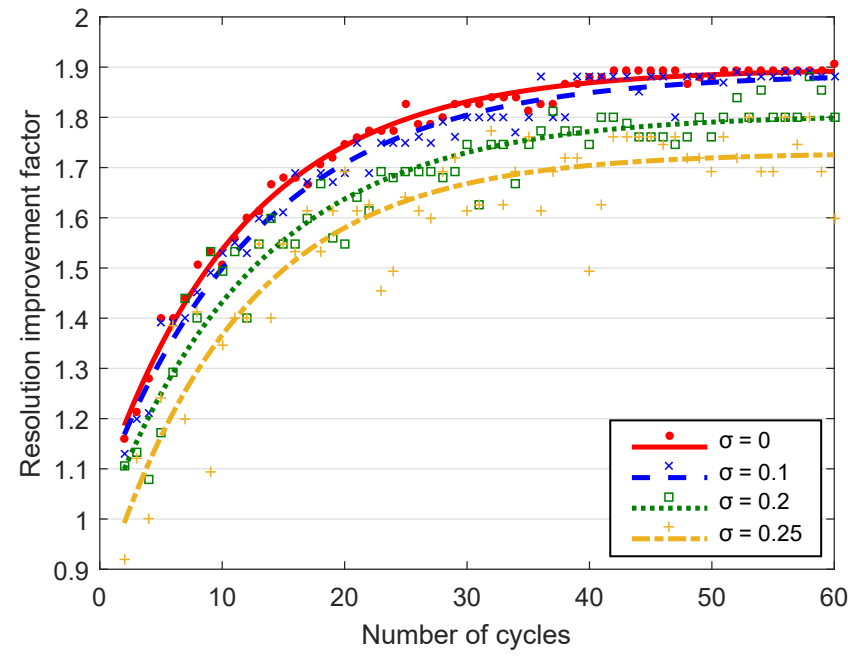

Fig. 3. Our simulations reconstructing a temporal chirp signal (with a $2 \times$ reconstruction factor, $p / q=2$ ) show that, relative to the number of cycles used for the reconstruction, the resolution improvement follows an exponential rise to a maximum limit of approximately $1.9 \times$ when the registration is perfect ( $\sigma=0$ frames). In the presence of zero-mean, normally distributed registration error with a standard deviation of $\sigma$, the resolution improvement also follows an exponential rise, but to a lower maximum limit.

shifts $w[n]$ to be known (no registration error, $\sigma=0$ ) and uniformly distributed over one frame interval. We measured resolution improvement by comparing the bandwidth of the reconstructed signal (with a $2 \times$ reconstruction factor, $p / q=2$ ) to the bandwidth of the observed, low-resolution signal, and we repeated the simulation using up to 60 cycles for the reconstruction. As expected, we found that the resolution improvement increases significantly as more cycles are included, but eventually, including additional cycles provides negligible improvement and is limited to a resolution improvement factor of approximately $1.9 \times$ when $p / q=2$ for a $2 \times$ reconstruction factor (Figure 3, $\sigma=0$ curve, see further description in Section III-B).

\section{B. Influence of Registration Error}

The performance of the sub-frame temporal registration step is critical to the superresolution reconstruction quality. Since sub-resolution registration accuracy is difficult to measure in practice (due to a lack of a ground truth in experimental data), we conducted a simulation on synthetic data to quantify the registration error. We generated a reference signal, $\hat{I}_{\mathrm{ref}}[n]=\cos (2 \pi f(n \Delta T))$, and a warped test signal, $\hat{I}[n]=\cos (2 \pi f(n \Delta T+\varepsilon[n]))$, where $\varepsilon[n]$ is a random shift drawn from a uniform distribution between $-\Delta T / 2$ and $\Delta T / 2$, $f$ is the signal frequency, and $\Delta T$ is the sample spacing. For consistency with our in vivo imaging experiments in which we image the zebrafish heart (beating at approximately 3 beats per second) at 30 frames per second, we chose $f=3 \mathrm{~Hz}$ and $\Delta T=1 / 30 \mathrm{~s}$. We registered the test signal $\hat{I}[n]$ to the reference signal $\hat{I}_{\text {ref }}[n]$ and compared the registered time indices to the true time indices to determine the registration error. In this simulation, for a sample size of 1000 time points, we observed that $80 \%$ of registered samples are within \pm 0.02 frames of the true temporal shift.

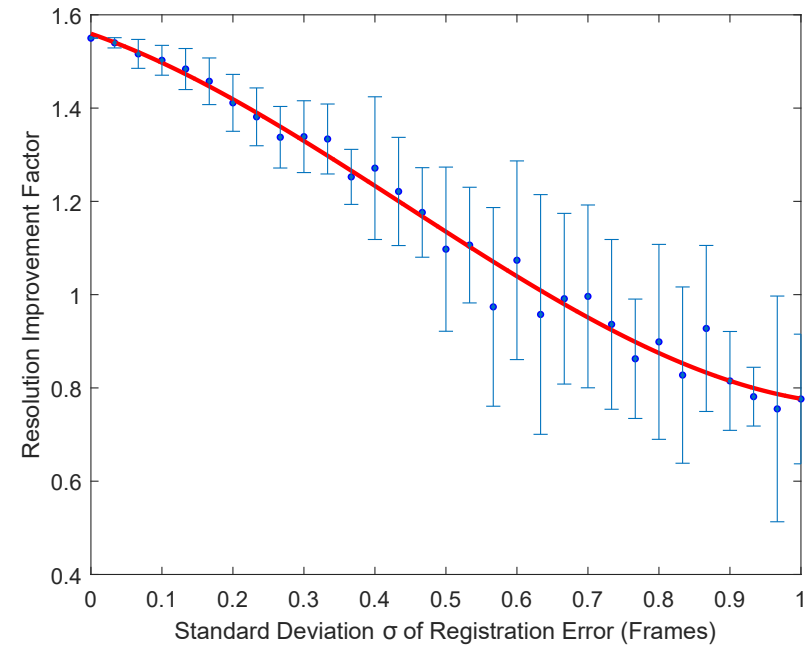

Fig. 4. We reconstruct a temporal chirp with a $2 \times$ reconstruction factor $(p / q=$ 2 ) from 10 cycles under different levels of registration error $\sigma$. As the standard deviation of the registration error increases, the resolution improvement in the reconstructed signal decreases. Beyond a standard deviation $\sigma=0.5$ frames, the reconstructed resolution is worse than the original resolution (resolution improvement factor $<1 \times)$.

We next performed a simulation to characterize the effect of registration error on the resolution improvement in the reconstruction. We generated 10 cycles of a repeating temporal chirp signal with a known sample index $w[n]$ at each sample. We then low-pass filtered and downsampled the signal by a factor of 2, and performed the reconstruction using incorrect sample indices, $w[n]+\varepsilon[n]$, where $\varepsilon[n]$ is a random error drawn from a zero-mean normal distribution with a standard deviation $\sigma$. We repeated this with various values of $\sigma$, and for each value of $\sigma$, we repeated this simulation 8 times. As $\sigma$ increases, the resolution improvement decreases, and past $\sigma=0.5$ frames, the resolution of the reconstruction is worse than the original resolution due to the registration step matching dissimilar samples together (Figure 4). We then repeated the simulation in which we varied the number of cycles used for reconstruction, this time imposing a random registration error to each sample, drawn from a normal distribution with standard deviation $\sigma=0.1,0.2$, and 0.25 . As we increase the number of cycles, the resolution improvement still increases, but the maximum resolution improvement limit decreases as $\sigma$ increases (Figure 3). We can use this result to estimate the registration error in the experimental data used in Figure 2. Since we used 14 cycles in our reconstruction in Figure 2, and we observed a resolution improvement of approximately $1.6 \times$, we estimate our registration error to have a standard deviation of approximately 0.1 frames.

\section{Comparison to Other Approaches}

In most live imaging scenarios, the acquisition frame rate is limited by the hardware of the camera system. These frame rate limits are set by the camera manufacturer based on the sensor technology, pixel readout time, and data transfer/storage time. For example, the Hamamatsu ImageEM C9100-13 EMCCD camera which we used in our experiments is limited to a maximum frame rate of approximately 30 frames per 
second at full resolution. Two common ways to achieve higher frame rates are through pixel binning and ROI (region of interest) selection. These are both common features on many commercial camera systems. In both cases, the number of pixels per image decreases, allowing the camera to acquire more frames per second. However, pixel binning reduces spatial resolution and ROI selection reduces the field of view. With our temporal superresolution method, we are able to achieve a higher effective frame rate without these disadvantages, as we demonstrate in Figure 5 and Figure 6 with fluorescent images of the beating heart in an embryonic $T g$ (cmlc2:eGFP) zebrafish [34] at 2 days post fertilization (dpf). In Figure 5, we acquired one image sequence at 60 frames per second with $2 \times$ spatial binning in both $x$ and $y$ dimensions, and we acquired a second image sequence at 30 frames per second, with $512 \times 512$ pixels, and without pixel binning. We then used the $30 \mathrm{fps}$ sequence (which spanned 12 cardiac cycles) to reconstruct a single cardiac cycle with a $2 \times$ temporal magnification factor using our algorithm. In Figure 6, we again show an image from this temporal superresolution reconstruction alongside an image from a sequence acquired at 60 frames per second using a $256 \times 256$ region of interest. In both figures, the $T g(\mathrm{cmlc2}: e G F P)$ zebrafish heart was imaged in fluorescence using a Leica DMI6000B inverted microscope with an HCX PL FLUOTAR L 40x/0.60 air objective and a Hamamatsu ImageEM C9100-13 EM-CCD camera. The full cardiac cycle corresponding to Figure 5 and Figure 6 are shown in supplementary movie 2 and supplementary movie 3 , respectively.

An important aspect in our method is the $\ell_{1}$ norm in the minimization function, Equation (16). Minimizing an $\ell_{1}$ norm is more computationally expensive than minimizing an $\ell_{2}$ norm (for a least-squares solution). The latter is much easier to implement and faster to compute, but the solution is more affected by outlier data. Outlier data can be caused by the shot noise typical in low-light fluorescence microscopy, irregularity in the repeating signal (such as arrhythmia), or registration error. We compared our proposed $\ell_{1}$ reconstruction with the corresponding least-squares reconstruction (i.e. the solution to Equation (16), except with $\ell_{2}$ norms replacing both of the $\ell_{1}$ norms) and with the result of temporal interpolation to evaluate the reconstructed image quality for both on-sample and offsample time points. Specifically, we used both $\ell_{1}$ and $\ell_{2}$ norms to reconstruct the cardiac cycle of a three day-old (three days post-fertilization) $G t(\text { tpm4-citrine })^{c t 31 a}$ zebrafish embryo [35] from a low temporal resolution sequence acquired at 30 frames per second [23]. We chose $\lambda$ for the $\ell_{1}$ reconstruction according to Equation (23), and we chose $\lambda$ for the leastsquares reconstruction to provide a similar background noise level as that of the $\ell_{1}$ reconstruction. We compared this to both cubic interpolation and motion interpolation (using Adobe After Effects [36]). Results show that our proposed method is best able to reduce motion blur (Figure 7). In fact, results show that the least-squares approach tends to over-smooth the solution, which can worsen the problem of motion blur. Additionally, while interpolation methods can be used to create higher rate sequences, they do not actually improve the effective temporal resolution, and they cannot
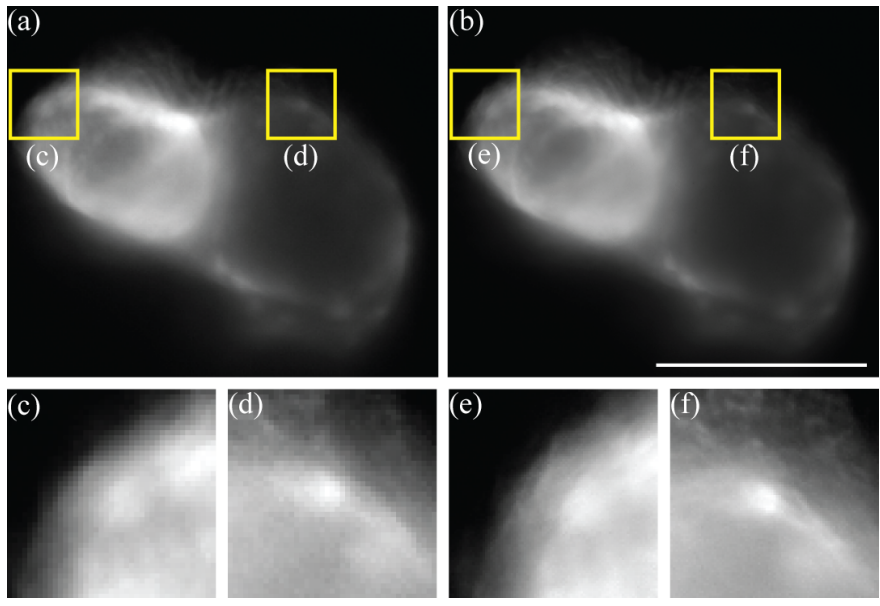

Fig. 5. The cardiac cycle of a live $T g(c m l c 2: e G F P)$ zebrafish is imaged in fluorescence with a $40 \times$ objective and a Hamamatsu C9100-13 EM-CCD camera, which has a maximum frame rate of 30 frames per second at full resolution. (a) $2 \times$ pixel binning allows us to acquire images at 60 frames per second, although with decreased spatial resolution $(256 \times 256$ pixels $)$. (b) Our temporal superresolution method is able to reconstruct a sequence with 60 frames per second at full spatial resolution $(512 \times 512$ pixels $)$ by combining twelve cardiac cycle sequences acquired at 30 frames per second. (c-f) Zoomed in regions show the loss in spatial resolution with pixel binning as compared to our method. Scalebar is $100 \mu \mathrm{m}$. See supplementary movie 2 for the full cardiac cycle.
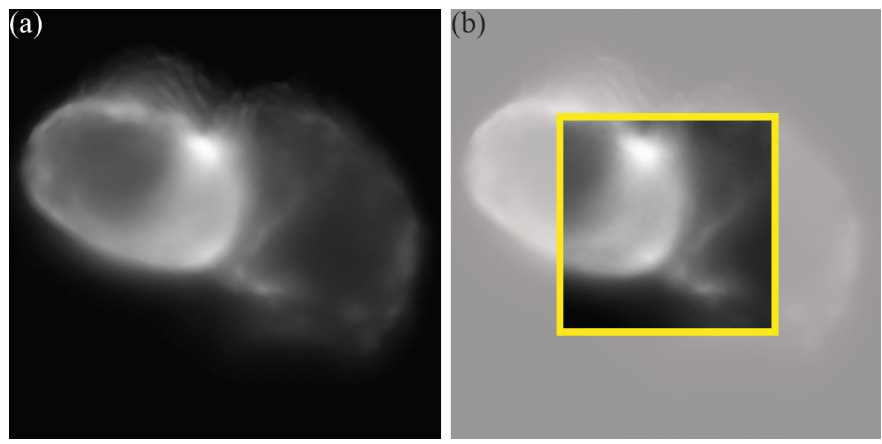

Fig. 6. (a) Using the same setup as used for Fig 5, our temporal superresolution method is able to reconstruct a sequence with 60 frames per second at full field $(512 \times 512$ pixels $)$ by combining twelve cardiac cycle sequences acquired at 30 frames per second. (b) Selecting a $256 \times 256$ region of interest allows us to acquire images at 60 frames per second, although with only a quarter of the field of view (central boxed region). The full field image $(512 \times 512$ pixels) is shown in the background for reference. See supplementary movie 3 for the full cardiac cycle.

reduce temporal aliasing or motion blur. Naive interpolation (using linear or cubic interpolation) can introduce additional spatial blur as a result of blending neighboring frames. Motion interpolation can avoid these frame-blending artifacts when calculating intermediate frames, but does not improve the resolution of frames that fall on integer samples. Our method is able to reconstruct a true higher rate sequence without additional spatial blurring in intermediate frames and with reduced motion blur in both on-sample and off-sample frames (Figure 7, Supplementary movie 4).

\section{3D Cardiac Reconstruction}

We applied our method to reconstruct a 3D time series of a beating heart in a live $\operatorname{Tg}(c m l c 2: e G F P)$ zebrafish embryo (at 

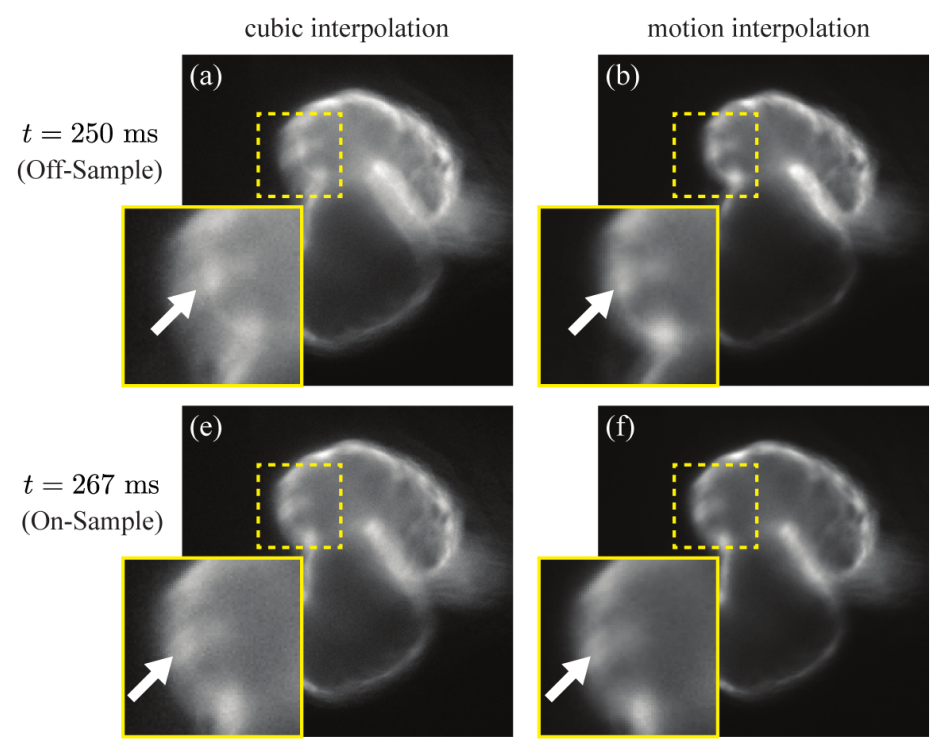
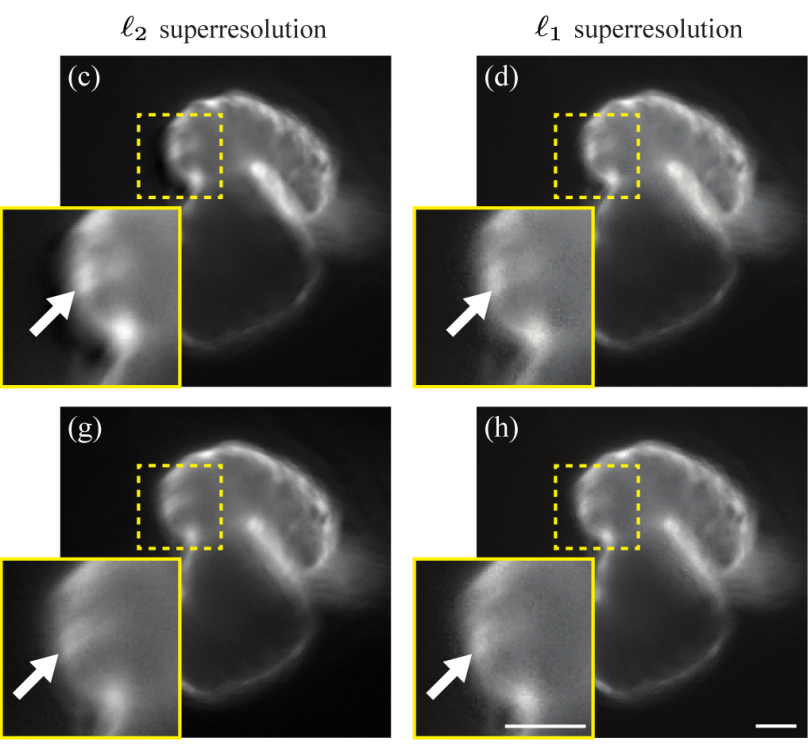

Fig. 7. The cardiac cycle of a live fluorescent Gt(tpm4-citrine) $)^{c t 3 l a}$ zebrafish embryo ( $\left.3 \mathrm{dpf}\right)$ is imaged at 30 fps with a $20 \times$ objective. (a-d) An off-sample frame halfway in between two original video frames (between $t=233 \mathrm{~ms}$ and $t=267 \mathrm{~ms}$ ) and (e-h) its neighboring on-sample frame (at $t=267 \mathrm{ms)}$ are reconstructed by $(\mathrm{a}, \mathrm{e})$ cubic interpolation, $(\mathrm{b}, \mathrm{f})$ motion interpolation using Adobe After Effects [36], (c,g) minimizing an $\ell_{2}$ version of our cost function with $\lambda=0.1$, and $(\mathrm{d}, \mathrm{h})$ minimizing our proposed $\ell_{1}$ cost function with $\lambda=0.15$. These choices for $\lambda$ produce similar background noise levels in both the $\ell_{1}$ and $\ell_{2}$ reconstructions. Arrows denote a bright region in the heart wall that is blurred due to the motion of the heart. A comparison of this region in each reconstruction shows that our proposed $\ell_{1}$ method is best at reducing this motion blur, both for off-sample and on-sample frames. Scalebars are $50 \mu \mathrm{m}$.

2.5 days post-fertilization) expressing green fluorescent protein in the heart [34]. We acquired a dataset consisting of 125 z-slices (with $2 \mu \mathrm{m}$ spacing between adjacent slices) at 60 frames per second using a custom-built multiview selective plane illumination microscope with two illumination arms and two detection arms [30]. Using this setup, we illuminated the sample through both illumination arms with a 3- $\mu$ m thick light sheet produced by rapidly scanning a Cobolt MLD $488 \mathrm{~nm}$ laser beam through the sample. We acquired images from a single view with a Nikon CFI Apo LWD $25 \times / 1.1$ water dipping objective and a Hamamatsu ORCA-Flash 4.0 V2 camera. To reduce file size and speed up processing time, we downsampled the images in the $x$ and $y$ directions to 180 pixels $\times 180$ pixels. Prior to our temporal superresolution reconstruction, we synchronized the z-slices using the method in [27]. We used nine heartbeat cycles to reconstruct a temporal superresolution sequence with twice the original sampling rate and with regularization parameter $\lambda=0.5$. In addition to temporal superresolution, our method also reduces noise, as shown in Figure 8 and supplementary movie 5.

To quantify the noise reduction of our temporal superresolution method, we calculated the peak signal-to-noise ratio (SNR), PSNR $=10 \log _{10}\left[\max \left(I^{2}\right) / \sigma^{2}\right]$, and the contrast-tonoise ratio $(\mathrm{CNR}), \mathrm{CNR}=10 \log _{10}\left[\left(\mu_{1}-\mu_{2}\right) / \sigma\right]$, where $I$ is the entire $3 \mathrm{D}+$ time image sequence, $\sigma$ is the standard deviation of a background region of the image sequence, and $\mu_{1}$ and $\mu_{2}$ are, respectively, the mean pixel intensities in appropriately chosen heart and background regions of interest. As shown in Table I, our method performs better than the wavelet-based PURE-LET method (which specifically takes into account Poisson-type noise, yet does not take advantage of temporal redundancy) [38] and our previous multicycle method [37] at denoising the experimental cardiac fluorescence image sequences.

TABLE I

DENOISING COMPARISONS FOR DATA IN FIGURE 8.

\begin{tabular}{|c|c|c|}
\hline & SNR [dB] & CNR [dB] \\
\hline Raw Synchronized & 38.17 & 12.59 \\
Multicycle Median Denoising [37] & 43.64 & 15.17 \\
PURE-LET Denoising [38] & 43.86 & 15.80 \\
Temporal Superresolution & $\mathbf{4 4 . 1 0}$ & $\mathbf{1 5 . 9 3}$ \\
\hline
\end{tabular}

\section{Discussion}

Improvements in this paper over our preliminary work [23] include a sub-frame temporal registration method that relaxes the requirement for perfectly periodic motions, and a procedure for determining the regularization parameter based on the L-curve [29]. Additionally, we demonstrate application in 4D $(3 \mathrm{D}+$ time) fluorescence imaging of the beating heart in a live zebrafish embryo (Figure 8). Our software, in the form of a Fiji plugin, is available online for download at [25]. For distribution purposes, this version uses an iterative reweighted least squares algorithm to solve the $\ell_{1}$ minimization [39] rather than the commercial IBM CPLEX package used in Section III. In terms of quality, both packages produce similar results (to within $1 \%$ ); however, CPLEX was up to $2.5 \times$ as fast as our reweighted least squares implementation on our setup.

We conclude by discussing some general features of the algorithm, in particular to answer the question: how many lowresolution cycles are necessary for a desired temporal superresolution factor? For 2D spatial superresolution with equally spaced sub-resolution shifts, if the reconstruction factor in each 

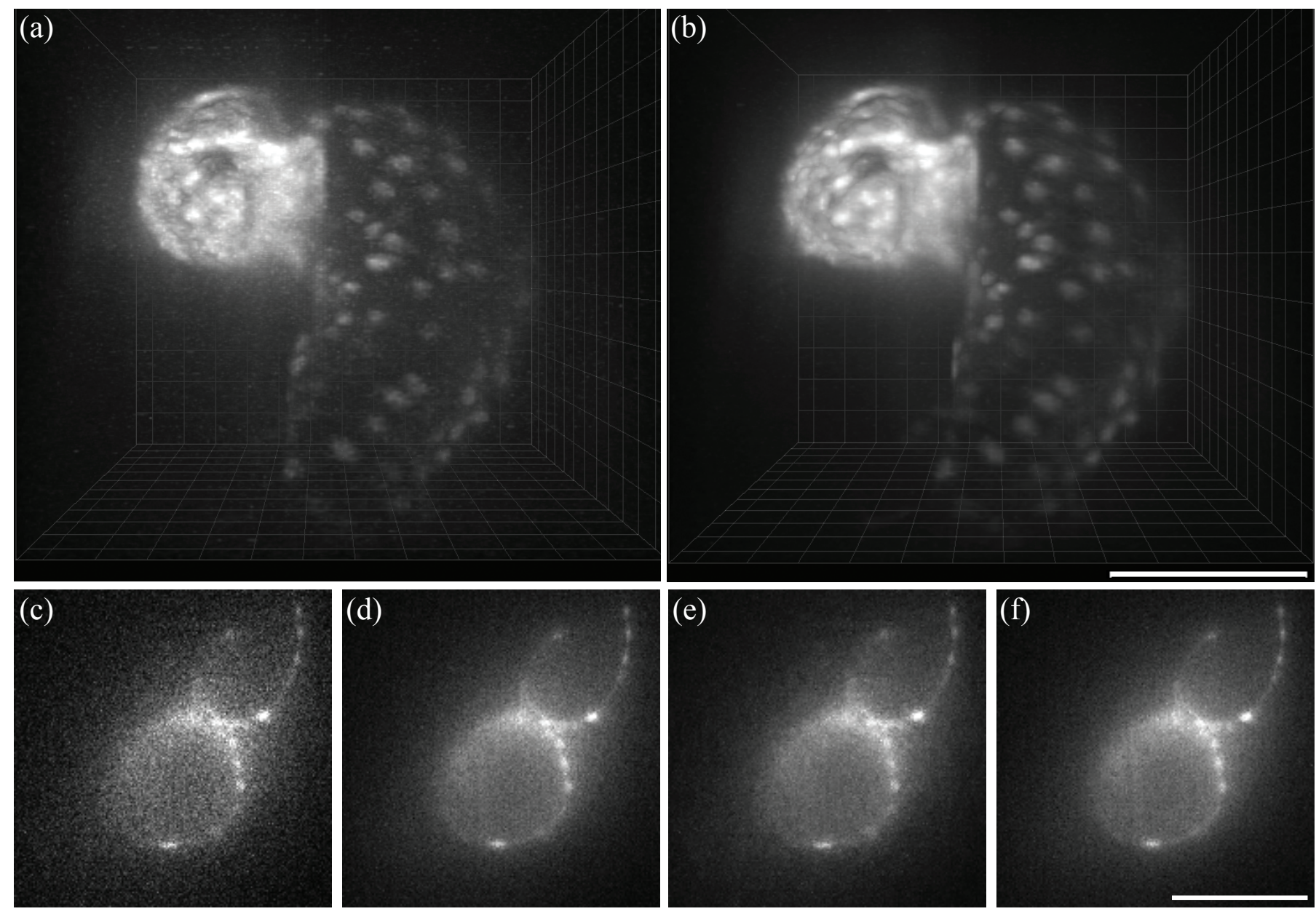

Fig. 8. We imaged the heart of a live, $2.5 \mathrm{dpf}, \operatorname{Tg}(\mathrm{cmlc2}$ :eGFP) zebrafish embryo in fluorescence at 60 frames per second. (a) $125 \mathrm{z}$-slices are synchronized to reconstruct a 3D volume. Due to the low illumination intensity and the short integration time used during acquisition, the resulting image is severely corrupted by Poisson-type noise. (b) Our temporal superresolution reconstruction is able to simultaneously temporally superresolve the image sequence and remove much of the noise. We used nine cardiac cycles with a regularization parameter $\lambda=0.5$ to reconstruct a single denoised heart beat with an effective sampling rate of 120 frames per second. See supplementary movie 5 for the full video showing the cardiac cycle. (c) An image with low signal-to-noise ratio from a single timepoint and $z$-slice is shown from the original image sequence. (d) The image is denoised using the multicycle denoising method in [37]. (e) The image is denoised using PURE-LET denoising [38]. (f) The corresponding denoised image is shown from our temporal superresolution reconstruction with $\lambda=0.5$. Quantitative denoising results are tabulated in Table I. Scale bar is $100 \mu \mathrm{m}$.

dimension $\mathcal{M}$ is an integer, the number of necessary lowresolution images has been shown to be $K=\mathcal{M}^{2}$ [33], [40]. It follows that for one-dimensional temporal superresolution, if the reconstruction factor, $\mathcal{M}=\frac{p}{q}$, is an integer, one should be able to expect the number of necessary low-resolution cycles to be $K=\mathcal{M}$. However, when the sub-integer shifts, $\Delta_{n}$, of the low-resolution cycles are randomly distributed over the interval $\left[0, N_{\text {int }}\right]$, using additional cycles improves results by making the problem overdetermined [40]. Since the subinteger shift is dependent on the phase of the underlying signal relative to the camera sampling rate, and since we do not gate our acquisitions, this sub-integer shift is not within our control. As a result, we often require $K>\mathcal{M}$ low-resolution cycles for reconstruction. The exact number of low-resolution cycles necessary will depend on the signal content, noise level, reconstruction factor $\mathcal{M}$, and the desired reconstruction accuracy [40]. Though there is no strict rule for the required number of low-resolution cycles, in our experiments we follow the guideline of having at least four times as many low- resolution cycles as our reconstruction factor, $K \geq 4 \mathcal{M}$. It is also important to note that a reconstruction factor of $\mathcal{M}$ does not necessarily correspond to a superresolution factor of $\mathcal{M}$. For example, we have shown in Figure 3 that, on synthetic data with no registration error and no signal-intrinsic variations, the resolution improvement is limited to approximately $1.9 \times$ for a reconstruction factor of $2 \times(p / q=2)$. While a larger reconstruction factor may continue to improve resolution beyond this limit, it is important to note that the condition number of the system matrix grows rapidly with respect to the reconstruction factor. Furthermore, a larger reconstruction factor leads to a more unstable inverse problem which requires heavier regularization to constrain the reconstruction to be smooth [41]. As a result, in practice we focus on superresolution with a $2 \times$ reconstruction factor.

We anticipate our method to be useful in cardiac development studies as a preprocessing step for algorithms such as cell segmentation and cell tracking, which perform more accurately with higher SNR and higher temporal resolution. In addition 
to cardiac imaging, we also anticipate our method to be useful for imaging other repeating biological processes such as muscle contractions, repeating depolarization waves (calcium imaging), and motion of individual cilia. In the presence of global sample motion (e.g. drift), it will be necessary to perform spatial registration as a pre-processing step in addition to temporal registration [42].

In summary, live fluorescence microscopy is often a difficult setting for quantitative imaging, particularly when imaging rapid dynamic samples. The tradeoff between spatio-temporal resolution and signal-to-noise ratio often results in images that are unsatisfactory in one aspect or the other (or both). Our method improves both without any additional hardware or hardware modifications. We achieve denoising by combining multiple cycles of the signal through the $\ell_{1}$ minimization in Equation (16). Additionally, this minimization also uses these multiple cycles to simultaneously achieve temporal superresolution (by a factor of 1.6 in experimental data), which is not possible with standard denoising algorithms. While our method is limited to repeating processes such as the cardiac cycle, we do not require (or assume) the process to be strictly periodic, as our method handles nonuniform temporal warping in the repeating process.

\section{APPENDIX A \\ REVERSE CUBIC INTERPOLATION FOR SUB-SAMPLE SHIFT ESTIMATION}

Rather than using a piecewise linear approximation to solve for the sub-sample shift $\Delta_{n}$ in Section II-B, in practice it is preferable to use a higher order approximation, such as a monotone cubic approximation [43], in which

$$
\begin{aligned}
\hat{I}[\mathbf{x}, n] \approx & \hat{I}_{\text {ref }}[\mathbf{x}, \bar{w}[n]] H_{1}\left(\Delta_{n}\right)+ \\
& \hat{I}_{\text {ref }}[\mathbf{x}, \bar{w}[n+1]] H_{2}\left(\Delta_{n}\right)+ \\
& D[\mathbf{x}, \bar{w}[n]] H_{3}\left(\Delta_{n}\right)+ \\
& D[\mathbf{x}, \bar{w}[n+1]] H_{4}\left(\Delta_{n}\right),
\end{aligned}
$$

where $D[\mathbf{x}, \bar{w}[n]]$ is the temporal derivative of $\hat{I}_{\text {ref }}$ at $\bar{w}[n]$ (which we calculate using the method in [43]), and $H_{k}\left(\Delta_{n}\right), k=1, \ldots, 4$ are the cubic Hermite basis functions evaluated at $\Delta_{n}$. Assuming that $0<\Delta_{n}<1$,

$$
\begin{aligned}
& H_{1}\left(\Delta_{n}\right)=2 \Delta_{n}^{3}-3 \Delta_{n}^{2}+1 \\
& H_{2}\left(\Delta_{n}\right)=-2 \Delta_{n}^{3}+3 \Delta_{n}^{2} \\
& H_{3}\left(\Delta_{n}\right)=\Delta_{n}^{3}-2 \Delta_{n}^{2}+\Delta_{n} \\
& H_{4}\left(\Delta_{n}\right)=\Delta_{n}^{3}-\Delta_{n}^{2} .
\end{aligned}
$$

Given the image $\hat{I}[\mathbf{x}, n]$ and the whole-sample, integer shift $\bar{w}[n]$, we calculate $\Delta_{n}$ with the following minimization:

$$
\Delta_{n}=\underset{t}{\arg \min } \mathcal{C}(t)
$$

where

$$
\begin{aligned}
\mathcal{C}(t)=\sum_{\mathbf{x}} & \left(\hat{I}_{\text {ref }}[\mathbf{x}, \bar{w}[n]] H_{1}(t)\right. \\
& +\hat{I}_{\mathrm{ref}}[\mathbf{x}, \bar{w}[n+1]] H_{2}(t) \\
& +D[\mathbf{x}, \bar{w}[n]] H_{3}(t) \\
& +D[\mathbf{x}, \bar{w}[n+1]] H_{4}(t) \\
& -\hat{I}[\mathbf{x}, n])^{2}
\end{aligned}
$$

which is a minimization of a scalar polynomial function and can be solved by finding the roots of $\frac{\mathrm{d}}{\mathrm{d} t} \mathcal{C}(t)$.

\section{REFERENCES}

[1] S. W. Hell and J. Wichmann, "Breaking the diffraction resolution limit by stimulated emission: stimulated-emission-depletion fluorescence microscopy," Opt. Lett., vol. 19, no. 11, pp. 780-782, Jun 1994.

[2] T. A. Klar, S. Jakobs, M. Dyba, A. Egner, and S. W. Hell, "Fluorescence microscopy with diffraction resolution barrier broken by stimulated emission," Proc. Nat. Acad. Sc., vol. 97, no. 15, pp. 8206-8210, 2000.

[3] M. G. L. Gustafsson, "Surpassing the lateral resolution limit by a factor of two using structured illumination microscopy," J. Microsc., vol. 198, no. 2, pp. 82-87, 2000.

[4] M. G. L. Gustafsson, "Nonlinear structured-illumination microscopy: Wide-field fluorescence imaging with theoretically unlimited resolution," Proc. Nat. Acad. Sc., vol. 102, no. 37, pp. 13081-13 086, 2005.

[5] R. Heintzmann, H. Münch, and C. Cremer, "High-precision distance measurements in epifluorescent microscopy-simulation and experiment," Cell Vision, vol. 4, pp. 252-253, 1997.

[6] E. Betzig, G. H. Patterson, R. Sougrat, O. W. Lindwasser, S. Olenych, J. S. Bonifacino, M. W. Davidson, J. Lippincott-Schwartz, and H. F. Hess, "Imaging intracellular fluorescent proteins at nanometer resolution," Science, vol. 313, no. 5793, pp. 1642-1645, 2006.

[7] M. J. Rust, M. Bates, and X. Zhuang, "Sub-diffraction-limit imaging by stochastic optical reconstruction microscopy (storm)," Nature Methods, vol. 3, pp. 793-796, 2006.

[8] T. V. Truong, W. Supatto, D. S. Koos, J. M. Choi, and S. E. Fraser, "Deep and fast live imaging with two-photon scanned light-sheet microscopy," Nature Methods, vol. 8, no. 9, pp. 757-760, 2011.

[9] T. A. Planchon, L. Gao, D. E. Milkie, M. W. Davidson, J. A. Galbraith, C. G. Galbraith, and E. Betzig, "Rapid three-dimensional isotropic imaging of living cells using bessel beam plane illumination," Nature Methods, vol. 8, no. 5, pp. 417-423, 2011.

[10] S. A. Jones, S. H. Shim, J. He, and X. Zhuang, "Fast, three-dimensional super-resolution imaging of live cells," Nature Methods, vol. 8, no. 6, pp. 499-505, 2011.

[11] J. Vermot, S. E. Fraser, and M. Liebling, "Fast fluorescence microscopy for imaging the dynamics of embryonic development," HFSP J., vol. 2, no. 3, pp. 143-155, June 2008.

[12] G. Bub, M. Tecza, M. Helmes, P. Lee, and P. Kohl, "Temporal pixel multiplexing for simultaneous high-speed, high-resolution imaging," Nature Methods, vol. 7, no. 3, pp. 209-211, March 2010.

[13] D. Reddy, A. Veeraraghavan, and R. Chellappa, "P2c2: Programmable pixel compressive camera for high speed imaging," in 2011 IEEE Conf. Comput. Vis. Patt. Recog. (CVPR), June 2011, pp. 329-336.

[14] E. Shechtman, Y. Caspi, and M. Irani, "Space-time super-resolution," IEEE Trans. Patt. An. Mach. Intel., vol. 27, no. 4, pp. 531-545, April 2005.

[15] A. Agrawal, M. Gupta, A. Veeraraghavan, and S. G. Narasimhan, "Optimal coded sampling for temporal super-resolution," in 2010 IEEE Conf. Comput. Vis. Patt. Recog. (CVPR), June 2010, pp. 599-606.

[16] A. Veeraraghavan, D. Reddy, and R. Raskar, "Coded strobing photography: Compressive sensing of high speed periodic videos," IEEE Trans. Patt. An. Mach. Intel., vol. 33, no. 4, pp. 671-686, 2011.

[17] D. W. Staudt, J. Liu, K. S. Thorn, N. Stuurman, M. Liebling, and D. Y. R. Stainier, "High-resolution imaging of cardiomyocyte behavior reveals two distinct steps in ventricular trabeculation," Development, vol. 141, no. 3, pp. 585-593, 2014. 
[18] R. Dixit and R. Cyr, "Cell damage and reactive oxygen species production induced by fluorescence microscopy: effect on mitosis and guidelines for non-invasive fluorescence microscopy," Plant J., vol. 36, no. 2, pp. 280-290, 2003.

[19] G. H. Patterson and D. W. Piston, "Photobleaching in two-photon excitation microscopy," Biophysical Journal, vol. 78, no. 4, pp. 2159 $-2162,2000$

[20] F. Sroubek, G. Cristobal, and J. Flusser, "A unified approach to superresolution and multichannel blind deconvolution," IEEE Trans. Image Proces., vol. 16, no. 9, pp. 2322-2332, Sept. 2007.

[21] S. Farsiu, M. Robinson, M. Elad, and P. Milanfar, "Fast and robust multiframe super resolution," IEEE Trans. Image Proces., vol. 13, no. 10, pp. 1327-1344, Oct. 2004.

[22] M. Ben-Ezra, A. Zomet, and S. K. Nayar, "Video super-resolution using controlled subpixel detector shifts," IEEE Trans. Patt. An. Mach. Intel., vol. 27, no. 6, pp. 977-987, June 2005.

[23] K. Chan, L. A. Trinh, and M. Liebling, "A temporal superresolution method applied to low-light cardiac fluorescence microscopy," in 2013 Conference Record of the 4 $7^{\text {th }}$ Asilomar Conference on Signals, Systems, and Computers, Nov 2013.

[24] J. Schindelin, I. Arganda-Carreras, E. Frise, V. Kaynig, M. Longair, T. Pietzsch, S. Preibisch, C. Rueden, S. Saalfeld, B. Schmid, J.Y. Tinevez, D. J. White, V. Hartenstein, K. Eliceiri, P. Tomancak, and A. Cardona, "Fiji: an open-source platform for biological-image analysis," Nature Methods, vol. 9, no. 7, pp. 676-682, 2012.

[25] "http://sybil.ece.ucsb.edu/pages/temporalsuperresolution."

[26] H. Takeda, P. Milanfar, M. Protter, and M. Elad, "Super-resolution without explicit subpixel motion estimation," IEEE Trans. Image Proces., vol. 18, no. 9, pp. 1958-1975, Sept 2009.

[27] M. Liebling, J. Vermot, A. S. Forouhar, M. Gharib, M. E. Dickinson, and S. E. Fraser, "Nonuniform temporal alignment of slice sequences for four-dimensional imaging of cyclically deforming embryonic structures," in Proc. 3rd IEEE Int. Symp. Biomed. Imag. (ISBI'06), April, 2006, pp. 1156-1159.

[28] G. B. Dantzig, A. Orden, and P. Wolfe, "The generalized simplex method for minimizing a linear form under linear inequality restraints." Pacific J. Math., vol. 5, no. 2, pp. 183-195, 1955.

[29] P. Hansen, "Analysis of discrete ill-posed problems by means of the L-curve,” SIAM Review, vol. 34, no. 4, pp. 561-580, 1992.

[30] U. Krzic, S. Gunther, T. E. Saunders, S. J. Streichan, and L. Hufnagel, "Multiview light-sheet microscope for rapid in toto imaging," Nature Methods, vol. 9, no. 7, pp. 730-733, 2012.

[31] ILOG-CPLEX, "High-performance software for mathematical programming and optimization," http://www.ilog.com/products/cplex, 2005.

[32] M. Westerfield, The Zebrafish Book: A Guide for the Laboratory Use of Zebrafish (Brachydanio Rerio), 4th Edition. Univ. of Oregon Press, Eugene, 2000.

[33] Z. Lin and H. Y. Shum, "Fundamental limits of reconstruction-based superresolution algorithms under local translation," IEEE Trans. Patt. An. Mach. Intel., vol. 26, no. 1, pp. 83-97, Jan. 2004.

[34] C.-J. Huang, C.-T. Tu, C.-D. Hsiao, F.-J. Hsieh, and H.-J. Tsai, "Germline transmission of a myocardium-specific gfp transgene reveals critical regulatory elements in the cardiac myosin light chain 2 promoter of zebrafish," Developmental Dynamics, vol. 228, no. 1, pp. 30-40, 2003.

[35] L. A. Trinh, T. Hochgreb, M. Graham, D. Wu, F. Ruf-Zamojski, C. S. Jayasena, A. Saxena, R. Hawk, A. Gonzalez-Serricchio, A. Dixson, E. Chow, C. Gonzales, H. Y. Leung, I. Solomon, M. Bronner-Fraser, S. G. Megason, and S. E. Fraser, "A versatile gene trap to visualize and interrogate the function of the vertebrate proteome," Genes \& Development, vol. 25, no. 21, pp. 2306-2320, Nov 2011.

[36] Adobe Systems Inc., "Adobe After Effects CS5," http://www.adobe.com/ products/aftereffects.html, accessed Nov. 30, 2015.

[37] S. Bhat, I. V. Larina, K. V. Larin, M. E. Dickinson, and M. Liebling, "Multiple-cardiac-cycle noise reduction in dynamic optical coherence tomography of the embryonic heart and vasculature," Opt. Lett., vol. 34, no. 23, pp. 3704-3706, Dec 2009.

[38] F. Luisier, C. Vonesch, T. Blu, and M. Unser, "Fast interscale wavelet denoising of poisson-corrupted images," Signal Processing, vol. 90, no. 2, pp. 415-427, February 2010.

[39] I. Daubechies, R. DeVore, M. Fornasier, and C. S. Gntrk, "Iteratively reweighted least squares minimization for sparse recovery," Comm. Pure \& Appl. Math., vol. 63, no. 1, pp. 1-38, 2010.

[40] D. Robinson and P. Milanfar, "Statistical performance analysis of superresolution," IEEE Trans. Image Proces., vol. 15, no. 6, pp. 1413-1428, June 2006.
[41] S. Baker and T. Kanade, "Limits on super-resolution and how to break them," IEEE Trans. Patt. An. Mach. Intel., vol. 24, no. 9, pp. 1167-1183, Sep. 2002.

[42] J. Yoo, I. V. Larina, K. V. Larin, M. E. Dickinson, and M. Liebling, "Increasing the field-of-view of dynamic cardiac OCT via post-acquisition mosaicing without affecting frame-rate or spatial resolution," Biomed. Opt. Express, vol. 2, no. 9, pp. 2614-2622, Sep 2011.

[43] F. N. Fritsch and R. E. Carlson, "Monotone piecewise cubic interpolation," SIAM J. Num. Analys., vol. 17, no. 2, pp. 238-246, 1980.

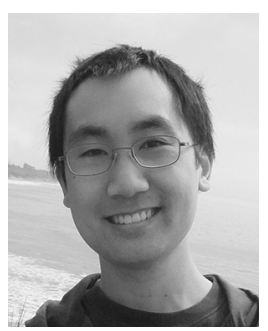

Kevin G. Chan (S'15) received the M.S. degree in Electrical and Computer Engineering from the University of California, Santa Barbara in 2013 and the B.S. degree in Engineering from Harvey Mudd College, Claremont, California in 2011. From 2015 to 2016, he worked in the Computational Bioimaging Group at Idiap Research Institute, Martigny, Switzerland. His research interests include computational imaging, inverse problems, and applications to fluorescence microscopy.

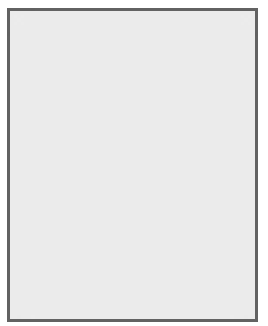

Sebastian J. Streichan is with the Kavli Institute for Theoretical Physics (KITP) at the University of California, Santa Barbara (UCSB).

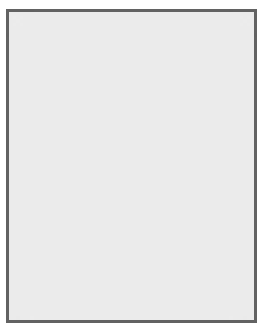

Le A. Trinh is a Senior Research Scientist at the University of Southern California in the department of Molecular and Computational Biology. She received her Ph.D. in Genetic and Developmental Biology from the University of California, San Francisco. Her research interests include developing molecular and genetic tools to enable the integration of embryonic development through optical imaging and genomics.

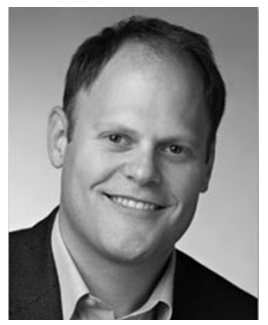

Michael Liebling (S'00-M'04) received M.S. (physics, 2000) and Ph.D. (2000) degrees from Ecole Polytechnique Fédérale de Lausanne, Lausanne, Switzerland. From 2004-2007, he was a post-doctoral scholar at the California Institute of Technology. In 2007, he joined the faculty of the Department of Electrical and Computer Engineering at the University of California, Santa Barbara, first as an assistant professor and, since July 2013, as associate professor. In 2015, he joined the Idiap Research Institute in Martigny, Switzerland as senior researcher, where he leads the computational bioimaging group.

His research interests include biological microscopy and image processing for the study of dynamic biological processes. Dr. Liebling is a member of the IEEE Signal Processing Society's Bio-Imaging and Signal Processing Technical Committee (BISP-TC), which he chaired in 2014-2015. He serves as Associate Editor (2015-2016) and Senior Associate Editor for IEEE Signal Processing Letters (2016-2018) and was Technical Program co-Chair of the IEEE International Symposium on Biomedical Imaging in 2011 and 2013 (ISBI'11, ISBI'13). 